\title{
Diskriminierungen vermeiden, Beschwerden ernst nehmen
}

\author{
Thomas Mäule
}

Im August 2006 ist in Deutschland das Allgemeine Gleichbehandlungsgesetz (AGG) in Kraft getreten. Arbeitgeber haben die Pflicht, die Antidiskriminierungsvorschriften zu beachten und in die betriebliche Praxis umzusetzen. Damit ergeben sich eine Reihe einrichtungsspezifischer Management-Herausforderungen. Das neue Gesetz kann auch Anlass sein, über die »Beschwerdekultur « einer Organisation nachzudenken.

Nach der zweiten und dritten Lesung im Bundestag ist das Allgemeine Gleichbehandlungsgesetz (AGG) umgangssprachlich auch Antidiskriminierungsgesetz genannt - seit 18 . August 2006 in Kraft. (1) Mit dem Allgemeinen Gleichbehandlungsgesetz werden vier EU-Richtlinien (2) zum Schutz vor Diskriminierung umgesetzt der bestehende Schutz gegen Benachteiligungen erheblich erweitert.

Schwerpunkt des Allgemeinen Gleichbehandlungsgesetzes ist der Schutz vor Diskriminierungen im Zusammenhang mit der Beschäftigung (§ 2 Abs. 1 AGG). Geschützt sind beispielsweise Mitarbeiterinnen und Mitarbeiter, Auszubildende, Bewerberinnen und Bewerber und ausgeschiedene Beschäftigte. Verboten sind Diskriminierungen wegen Rasse oder ethnischer Herkunft, Geschlecht, Religion oder Weltanschauung, Behinderung, Alter sowie sexueller Identität (§ 1 AGG). Das Gesetz verlangt zum einen vom Dienstgeber, selbst niemanden zu diskriminieren. Zum anderen muss die Einrichtung dafür Sorge tragen, dass die Beschäftigten vor Diskriminierung durch andere Beschäftigte oder Dritte (also auch Kunden und Klienten) geschützt sind (§ 12 AGG) (3).

Unter Diskriminierung versteht das Gesetz unmittelbare oder mittelbare
Benachteiligungen sowie allgemeine und sexuelle Belästigungen ( $§ 3$, Abs. 1 bis 4). Verboten ist nicht jede Form der Benachteiligung. Zulässig sind Ungleichbehandlungen, die gerechtfertigt sind (beispielsweise aufgrund unterschiedlicher Leistung oder Vorbildung, vgl. § 8 Abs. 1). An die Rechtfertigungsgründe wird jedoch ein strenger Maßstab angelegt.

Die »Kirchenklausel « (§ 9 AGG) ist so ausgestaltet, dass dem Selbstbestimmungsrecht der Kirchen und der ihnen zugeordneten Einrichtungen (z. B. Caritas, Diakonie) Rechnung getragen wird. Das bedeutet, dass kirchliche Einrichtungen ihre Beschäftigten weiterhin mit Rücksicht auf deren Religion oder Weltanschauung auswählen dürfen. Eine unterschiedliche Behandlung ist erlaubt, wenn eine bestimmte Religion oder Weltanschauung (»christliche Werte bzw. Haltungen «) eine gerechtfertigte berufliche Anforderung darstellt ( $\$ 9$ Abs. 1 AGG) (4). Außerdem kann von den Beschäftigten ein loyales und aufrichtiges Verhalten im Sinne des Selbstverständnisses der Kirche verlangt werden (§ 9 Abs. 2 AGG).

\section{Rechte für die Mitarbeitenden}

Aus dem Allgemeinen Gleichbehandlungsgesetz ergeben sich für die betroffenen Mitarbeitenden folgende Rechte:

- Jede Beschäftigte und jeder Beschäftigte hat nach $\S 1$ des Allgemeinen Gleichbehandlungsgesetzes das Recht, sich bei einer zuständigen Stelle der Einrichtung zu beschweren, wenn er oder sie sich im Zusammenhang mit dem Dienstverhältnis vom Dienstgeber, von Vorgesetzten, anderen Beschäftigen oder Dritten wegen eines Benachteiligungsgrundes ( $\$ 1$ AGG) diskriminiert fühlt.

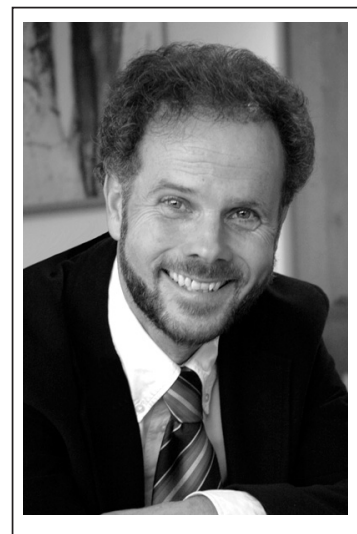
Dr. Tho-
mas Mäule studierte Theolo- gie, Sport- wissen- schaft, Geronto- logie und Sozial- management. Er war Gemeindepfarrer, Studienleiter an der Evangelischen Akademie Bad Boll und Leiter des Grundsatzreferates im Diakonischen Werk Württemberg. Derzeit ist er Referent für Theologie und Ethik bei der Evangelischen Heimstiftung e. V. in Stuttgart. Die Organisation ist der größte Anbieter von Altenhilfedienstleistungen in Baden-Württemberg. In 51 Alten- und Pflegeheimen arbeiten rund 6.000 Beschäftigte. E-Mail

t.maeule@ev-heimstiftung.de

- Falls der Dienstgeber keine oder offensichtlich ungeeignete Maßnahmen zur Unterbindung einer Benachteiligung oder (sexuellen) Belästigung am Arbeitsplatz ergreift, sind die betroffenen Mitarbeitenden nach § 14 Allgemeine Gleichbehandlungsgesetz berechtigt, ihre Tätigkeit ohne Verlust des Arbeitsentgelts einzustellen (bzw. zu verweigern, vgl. § 273 BGB) bzw. auf Unterlassung zu klagen (§ 1004 BGB).

- Die Mitarbeitenden haben aus $\S 12$ des Allgemeinen Gleichbehandlungsgesetzes einen Abhilfeanspruch gegen den Dienstgeber. 
- Nach § 15 des Allgemeinen Gleichbehandlungsgesetzes kann der Mitarbeitende bei einem Verstoß gegen das Benachteiligungsverbot einen Entschädigungs- bzw. Schadensersatzanspruch gegen den Dienstgeber haben. Diesen Anspruch muss er grundsätzlich innerhalb einer Frist von zwei Monaten schriftlich geltend machen.

\section{Organisationspflichten des Arbeitgebers}

Der Arbeitgeber ist nach dem Allgemeinen Gleichbehandlungsgesetz verpflichtet, in seinem Unternehmen die erforderlichen Maßnahmen zum Schutz der Beschäftigten vor Benachteiligungen zu schaffen. Dazu gehören auch präventive Maßnahmen (§ 12 Abs. 1 AGG).

- Nach § 13 ist der Arbeitgeber verpflichtet, eine für Diskriminierungsfragen zuständige Stelle (AGG-Beauftragte/r) einzurichten und das Gesetz sowie den Beauftragten in der Einrichtung bekannt zu machen (§ 12 Abs.5 AGG).

- Aus $\S 12$ Abs. 2 ergibt sich eine Pflicht zur Mitarbeiterschulung. Diese soll zur Vermeidung von Benachteiligung erfolgen.

- Nach § 22 besteht eine Beweislastumkehr zulasten des Dienstgebers. Dieser kann eine vermutete Diskriminierung nur dann wirksam widerlegen, wenn er seine Entscheidungen plausibel dokumentiert hat und durch Unterlagen oder Zeugen beweisen kann.

\section{Eine Herausforderung an das Management}

Welche organisatorischen Maßnahmen zum Schutz gegen Benachteiligung sind seitens des Arbeitgebers zu ergreifen? Welche neuen Managementaufgaben sind zu erfüllen, um Einrichtungen gesetzeskonform zu organisieren und so vor Schadensersatz- und Entschädigungsklagen zu schützen? Wenn im Folgenden Maßnahmen genannt werden, so werden diese - je nach Größe des Unternehmens - unterschiedlich zu beurteilen sein. Die Verpflichtung kann nur so weit gehen, wie der Arbeitgeber rechtlich und tatsächlich zur Pflichterfüllung in der Lage ist.
Zur Umsetzung des Allgemeinen Gleichbehandlungsgesetzes sind folgende Maßnahmen gefordert:

- Information und Schulung: Zunächst muss das Allgemeine Gleichbehandlungsgesetz durch Aushang oder mittels Einsatz der üblichen Informationstechniken bekannt gemacht werden. Als Information kann darüber hinaus (in Absprache mit Mitarbeitervertretung oder dem Betriebsrat) ein Merkblatt an alle Mitarbeitende versandt werden. Das Aushändigen (und Gegenzeichnen) eines Merkblattes mag wirkungsvoll sein, reicht aber als Pflichterfüllung nach § 12 Abs. 1 des Allgemeinen Gleichbehandlungsgesetzes und unter präventiven Gesichtspunkten (§ 1 AGG) nicht aus. Nach § 12 des Allgemeinen Gleichbehandlungsgesetzes erfüllen Träger und Einrichtungen durch Schulungen, in denen die Diskriminierungsverbote bekannt gemacht werden, ihre Pflicht des vorbeugenden Schutzes. In (Inhouse-) Seminaren wäre (zunächst) Führungskräften, Mitarbeitenden des Personalmanagements, der Mitarbeitervertretungen und (dann) allen Mitarbeitenden zu vermitteln, wie Diskriminierungen verhindert werden können und wie man sich dagegen wehren kann.

- Verhaltenskodex: Viele Einrichtungen haben bereits Leitbilder und daraus abgeleitete Handlungsgrundsätze. Das Thema »Benachteiligung « wird bislang kaum thematisiert. Hier bietet es sich an, den bestehenden Verhaltenskodex um entsprechende Antidiskriminierungsregeln zu ergänzen (um auch hier der nach $\S 12$ Abs. 2 Satz 2 des Allgemeinen Gleichbehandlungsgesetzes geforderten Organisationspflicht nachzukommen).

- Einrichten einer Beschwerdestelle: Nach § 13 des Allgemeinen Gleichbehandlungsgesetzes ist der Arbeitgeber verpflichtet, eine für Diskriminierungsfragen zuständige Stelle (AGG-Beauftragte/r) einzurichten. An die Beschwerdestelle können sich Personen richten, die sich diskriminiert fühlen. Die Beschwerden müssen dokumentiert, geprüft und das Ergebnis schriftlich mitgeteilt werden. Sollte eine Benachteiligung vorliegen, muss diese mit geeigneten Mitteln unterbunden werden. Als AGG-Beauftragte/r empfiehlt sich eine neutrale Person, die sowohl das Vertrauen der Führungs- kräfte als auch den Respekt der Mitarbeitenden genießt.

- Überprüfen von Verträgen und Vereinbarungen: Bei großen Trägern ist eine Überprüfung von Dienstverträgen, Dienstvereinbarungen, Personalauswahlverfahren etc. angezeigt. Interne Verfahren (z. B. für Fortbildung, Sonderurlaub) sind diskriminierungsfrei zu gestalten. Kriterienkataloge sind daraufhin zu sichten, ob unzulässige Kriterien angewandt werden.

- Überprüfung der Dokumentationspraxis: Laut Allgemeinen Gleichbehandlungsgesetzes besteht eine Beweislastumkehr zulasten des Dienstgebers. Eine vermutete Diskriminierung kann nur dann wirksam widerlegt werden, wenn die Entscheidungen plausibel dokumentiert und durch Unterlagen oder Zeugen zu beweisen sind. In Qualitätsmanagement-Handbüchern ist zu regeln, wie künftig beispielsweise bei Stellenausschreibungen und bei Absagen von Bewerbungen die Unterlagen (in Kopie) aufbewahrt, der Kriterienkatalog und Entscheidungsgründe dokumentiert werden. Zum Nachweis vorbeugender Schritte gehört auch, dass die Liste der Teilnehmenden bei Schulungen aufgehoben wird.

\section{Erweitertes \\ Konfliktmanagementsystem}

Weiterführend soll an dieser Stelle gefragt werden: Sind die beschriebenen Problemlösungen ausreichend? Oder ist es sinnvoll, über die Minimallösung hinaus ein erweitertes Konfliktmanagementsystem einzuführen, wenn eine Vielzahl von Mitarbeitenden ohnedies zu Schulungen zusammengerufen werden muss? Könnte das Allgemeine Gleichbehandlungsgesetz nicht eine einmalige Chance bieten, ein Beschwerdesystem im Unternehmen zu implementieren, das Konfliktkosten senkt und Arbeitszufriedenheit steigert?

Das neue Allgemeine Gleichbehandlungsgesetz wird relevant im Blick auf Beurteilungsgespräche, Vergütungsfragen, bei sensiblen Vorgängen im Personalwesen, der Behauptung sexueller Belästigung, Verstößen und Verletzungen wichtiger Rechtsgüter. Was wünschte man sich in all den Beschwerden mehr als den objek- 
tiven, neutralen und sachverständigen »Dritten«, der Verständnis für das jeweilige Anliegen zeigt?

Ein Medium hierfür ist ein neutrales und unabhängiges Beschwerdesystem. Bei jeder Beschwerde geht es um Macht und Interessen. Zwischen den Beteiligten besteht meist aber ein Machtgefälle und einseitige Abhängigkeiten. Hier hat eine unabhängige Stelle die besten Chancen, für alle Seiten akzeptable Lösungen zu entwickeln. Sie ist dabei nicht parteilich, vertritt aber konsequent die Interessen der Betroffenen. Sie hat keine Machtposition. Ihre Aufgabe besteht vielmehr darin, einen potenziellen Machtmissbrauch auszuschließen, zur Ausbalancierung von Macht beizutragen und so eine Balance zwischen Arbeitgeber und Arbeitnehmer zu schaffen.

Ein solches System setzt freilich voraus, dass beide Seiten sich auf das einlassen, was an - berechtigter und unberechtigter — Kritik, Beschwerden, an Wünschen und Vorschlägen artikuliert wird. Neben dem Aspekt der Zufriedenheit der Mitarbeiterinnen und Mitarbeiter können Beschwerden und Kritik im positiven Sinn als Prüfsteine für Qualität betrachtet werden und Ausgangspunkt für interne Leistungsverbesserung sein (vgl. Bucher 2004). Ein Beschwerdesystem kann darüber hinaus auch ein Frühwarnsystem bei etwaigen Missständen sein. Nach Titz und Paratsch übernimmt es »die Rolle einer roten Ampel« $(2001,26)$.

\section{Akzeptanz eines Beschwerdesystems}

Was aber sind Voraussetzungen für Akzeptanz und Funktionieren einer solchen Beschwerdestelle? Entscheidend ist:

- ein niedrigschwelliges System mit leichter Kontaktaufnahme (sei es persönlich, telefonisch oder schriftlich)

- die Zusicherung, dass Träger und Einrichtung die Arbeit der Stelle einerseits als legitime Einmischung akzeptieren und sie andererseits bereit sind, sich ernsthaft darauf einzulassen

- der deutliche Hinweis an Mitarbeitende, dass Beschwerden willkommen und erwünscht sind

- das Versprechen, dass jede Eingabe ernst genommen und den Kritikpunkten nachgegangen wird
- die Rückmeldung, dass das Anliegen verstanden wurde und innerhalb eines bestimmten Zeitrahmens ein Bescheid über das Ergebnis vorliegt

- die Integrität, denn die Beschwerdeinstanz entscheidet im Folgenden darüber, wer in welcher Form mit der Beschwerde konfrontiert wird.

Mit der Skizze dieser wenigen Bedingungen wird eine Beschwerdekultur deutlich, die klare Strukturen und Abläufe kennt, Transparenz ermöglicht und vorbehaltlos den Kritikpunkten nachgeht. Entscheidend sind Vertrauenswürdigkeit und Ernsthaftigkeit. Stauss und Seidel sehen dies »als Voraussetzung für die langfristige Überlebensfähigkeit eines Unternehmens « und werben dafür, »Beschwerden nicht primär als

\section{Anmerkungen}

(1) Bundesgesetzblatt. Jahrgang 2006. Teil I Nr. 39, ausgegeben zu Bonn am 17. August 2006, 1897-1910. Der Gesetzestext ist im Internet abrufbar unter http://www.bmj-bund.de.

(2) Die Europäische Union (EU) hat zum Schutz vor Diskriminierung Richtlinien erlassen. Hintergrund der europäischen Gesetzgebung ist der Gedanke, dass die Europäische Union nicht nur eine Wirtschafts-, sondern auch eine Wertegemeinschaft ist. Der Schutz vor Diskriminierung gehört zum Kernbestand der Menschenrechtspolitik. Ziel sozialer aber auch wirtschaftlicher - Überlegungen ist es, Minderheiten und benachteiligte Gruppen so weit als möglich zu integrieren. Die Bundesrepublik

Deutschland ist verpflichtet, die EURichtlinien (2000/43 EG, 2000/78 EG, 2002/73EG, 2004/113/EG) in innerstaatliches Recht zu transformieren. Diese Richtlinien verpflichten die EU-Mitgliedsstaaten, den Schutz vor Diskriminierung im Bereich Beschäftigung und Beruf hinsichtlich der Merkmale »Rasse, ethnische Herkunft«, »Religion, Weltanschauung «, »Behinderung «, »Alter «, »sexuelle Identität « und »Geschlecht« umzusetzen.

(3) Im Detail werden die Regelungen erläutert und mit zahlreichen Beispielen illustriert von Kock 2006; Wisskirchen 2006; Wüst 2006.

(4) Belling begründet diesen Freiraum mit der bisherigen Rechtsprechungspraxis und dem Selbstbestimmungsrecht der Kirchen aus Art. 137 III WRV i. V. mit Art. 140 GG und der Religionsfreiheit aus Art. 4 GG $(2004,888)$. Die in Anmerkung 2 zitierte europäische Richtlinie 2000/78/EG macht es den Mitgliedsstaaten möglich, »in Bezug auf berufliche Tätigkeiten innerhalb von Kirchen und anderen öffentlichen oder privaten Organisationen, deren Ethos auf religiösen abzuwehrendes Problem, sondern als Chance (zu) sehen « $(1998,18)$. Die Akzeptanz einer solchen Stelle hängt mit davon ab, ob sie mit den erforderlichen Kompetenzen ausgestattet ist, um auch in schwierigen Fällen verlässlich die Interessen der Ratsuchenden zu vertreten und Widerständen standhalten zu können.

\section{Fazit}

Eine konsequente Verbindung von fachlichen, prozessualen, kommunikativen und politischen Zielen ist mit der Einführung des Allgemeinen Gleichbehandlungsgesetzes verbunden - mit dem ethischen Anspruch: Beschäftigte vor Diskriminierung am Arbeitsplatz zu schützen.
Grundsätzen oder Weltanschauungen beruht, Bestimmungen (...) beizubehalten oder in künftigen Rechtsvorschriften Bestimmungen vorzusehen, die (...) bestehende einzelstaatliche Gepflogenheiten widerspiegeln und wonach eine Ungleichbehandlung wegen der Religion (...) keine Diskriminierung darstellt, wenn die Religion (...) eine wesentliche, rechtmäßige und gerechtfertigte berufliche Anforderung angesichts des Ethos der Organisation darstellt« (§ 4 Abs. 2).

\section{Literatur}

Belling, D. W.: Umsetzung der Antidiskriminierungsrichtlinie im Hinblick auf das kirchliche Arbeitsrecht. In: Neue Zeitschrift für Arbeitsrecht 21(2004)16, S.885-889.

Bucher, U.: Schwächen erkennen, Qualität sichern. Beschwerdemanagement. In: Sozialwirtschaft 14 (2004)1, S.28-30.

Kock, M.: Allgemeines Gleichbehandlungsgesetz. Überblick über die arbeitsrechtlichen Regelungen. In: Monatsschrift für Deutsches Recht. Zeitschrift für die Zivilrechtspraxis 19/2006, S. 1088-1093.

Stauss, B./Seidel, W.: Beschwerdemanagement. Fehler vermeiden - Leistung verbessern - Kunden binden. 2. Aufl., München/Wien 1998.

Titz, K./Paratsch, F.: Unser Ziel: Ihre Zufriedenheit. Gemeinsam mit den Bewohnern die Qualität verbessern. In: Altenheim, 40 (2001)10, S.26-29.

Wisskirchen, G.: Das Allgemeine Gleichbehandlungsgesetz. Auswirkungen auf die Praxis. 2. akt. u. erw. Aufl., Frechen 2006.

Wüst, H.: Allgemeines Gleichbehandlungsgesetz. Münster 2006. 